\title{
STABILIZATION OF OSCILLATORS SUBJECT TO DRY FRICTION: FINITE TIME CONVERGENCE VERSUS EXPONENTIAL DECAY RESULTS
}

\author{
ALEXANDRE CABOT
}

\begin{abstract}
We investigate the dynamics of an oscillator subject to dry friction via the following differential inclusion:

$$
\ddot{x}(t)+\partial \Phi(\dot{x}(t))+\nabla f(x(t)) \ni 0, \quad t \geq 0,
$$

where $f: \mathbb{R}^{n} \rightarrow \mathbb{R}$ is a smooth potential and $\Phi: \mathbb{R}^{n} \rightarrow \mathbb{R}$ is a convex function. The friction is modelized by the subdifferential term $-\partial \Phi(\dot{x})$. When $0 \in$ $\operatorname{int}(\partial \Phi(0))$ (dry friction condition), it was shown by Adly, Attouch, and Cabot (2006) that the unique solution to $(S)$ converges in a finite time toward an equilibrium state $x_{\infty}$ provided that $-\nabla f\left(x_{\infty}\right) \in \operatorname{int}(\partial \Phi(0))$. In this paper, we study the delicate case where the vector $-\nabla f\left(x_{\infty}\right)$ belongs to the boundary of the set $\partial \Phi(0)$. We prove that either the solution converges in a finite time or the speed of convergence is exponential. When $\Phi=a||+.b|\cdot|^{2} / 2, a>0$, $b \geq 0$, we obtain the existence of a critical coefficient $b_{c}>0$ below which every solution stabilizes in a finite time. It is also shown that the geometry of the set $\partial \Phi(0)$ plays a central role in the analysis.
\end{abstract}

\section{INTRODUCTION}

Consider a one-dimensional oscillator moving under the action of a recalling force. When the oscillator is subject to a constant Coulomb friction, it is wellknown that the dynamics stops definitively after a finite time. This type of result can be found in many monographs relative to differential equations ( $c f$. for example [13]). Many authors have investigated the stabilization of a coupled system of oscillators subject to Coulomb friction. Among them, Díaz and Millot [11] have studied a model in which each particle is connected to its neighbors by harmonic springs and the friction force is assumed to be of Coulomb type. The same dynamical system can also be derived from the spatial discretization of a vibrating string equation subject to solid friction. This is precisely the approach of Bamberger and Cabannes [5] who have proved that the equilibrium is reached after a finite time in the case of pure solid friction. The situation is more delicate in the presence of viscous friction. In this case, Díaz and Millot [11] point out a dichotomy phenomenon based on the relative importance of the viscous friction. When the viscous

Received by the editors December 15, 2004 and, in revised form, August 6, 2005.

2000 Mathematics Subject Classification. Primary 34C15, 34A60; Secondary 70F40, 37N05.

Key words and phrases. Differential inclusion, dry friction, nonlinear oscillator, finite time convergence, exponential decay, convex analysis. 
component is above some given threshold, the oscillators may not stabilize in a finite time.

Since the works of Moreau [15, 16, 14], it is well-known that convex analysis provides a satisfactory mathematical framework for the problems of contact in unilateral mechanics. In many situations involving dry contact, the friction force is modelized by a subdifferential operator (see for instance $[2,18]$ ). These considerations lead us to study the motion of an oscillator satisfying the following second-order in time differential inclusion

$$
\ddot{x}(t)+\partial \Phi(\dot{x}(t))+\nabla f(x(t)) \ni 0, \quad t \geq 0,
$$

where $\Phi: \mathbb{R}^{n} \rightarrow \mathbb{R}$ is a convex function and $f: \mathbb{R}^{n} \rightarrow \mathbb{R}$ is a smooth function. In this model, the external forces derive from the potential $f$. The possibly nonlinear term $\nabla f$ is aimed at covering the case of nonlinear oscillators. The formulation of the friction via the term $-\partial \Phi(\dot{x})$ allows us to generate a large variety of friction models. For example, the function $\Phi=\mid$.| corresponds to a Coulomb friction, while $\Phi=|.|^{2}$ gives a viscous one and the associated "Heavy Ball with Friction" system; cf. [4]. Notice also that the function $\Phi=|\cdot|^{p} \quad(p \in] 1,2[)$ generates an intermediate situation, which has been studied by Amann-Díaz [3] and Díaz-Liñán [10] under the terminology of "strong friction". The dynamical system $(S)$ provides us with a unifying framework, and most of the equations considered in $[5,11]$ can be viewed as particular cases of $(S)$. In our paper, we assume that the friction is dry, which is expressed by the condition $0 \in \operatorname{int}(\partial \Phi(0))$. Under this assumption, it is shown in [1] that the unique solution to $(S)$ converges to some equilibrium $x_{\infty} \in \mathbb{R}^{n}$ satisfying $-\nabla f\left(x_{\infty}\right) \in \partial \Phi(0)$. When moreover the vector $-\nabla f\left(x_{\infty}\right)$ belongs to the interior of the set $\partial \Phi(0)$, the authors prove that the dynamics stop definitively after a finite time. Counterexamples to finite time convergence exist when the vector $-\nabla f\left(x_{\infty}\right)$ belongs to the boundary of $\partial \Phi(0)$. The purpose of this paper is to analyse this delicate case and to evaluate the speed of convergence when finite time stabilization fails.

We show that the dynamics associated to $(S)$ may lead to two structurally different asymptotic behaviors, depending on the geometry of the set $\partial \Phi(0)$. When the set $\partial \Phi(0)$ is smooth (in a sense that will be described next), the velocity $\dot{x}(t)$ admits a limit direction (when $t \rightarrow+\infty$ ), which is normal to the set $\partial \Phi(0)$. On the other hand, when the set $\partial \Phi(0)$ is a convex polyhedron, the velocity vector $\dot{x}$ is shown to be normal to the set $\partial \Phi(0)$ after a finite time.

Another major point of the article lies in the estimation of the convergence rate. We prove that either the solution converges in a finite time or the speed of convergence is exponential. Our results are slightly more precise when the set $\partial \Phi(0)$ is smooth. We establish in this case that, if the excess of the set $\partial \Phi(x)$ over the set $\partial \Phi(0)$ tends to 0 sufficiently fast when $x \rightarrow 0$, then every solution to $(S)$ stabilizes in a finite time. When $\Phi=a|\cdot|+b|\cdot|^{2} / 2, a>0, b \geq 0$, we obtain the existence of a critical coefficient $b_{c}>0$ below which every solution stops definitively after a finite time.

Such results in finite dimension open interesting perspectives concerning similar results for mechanical and physical systems with infinite degrees of freedom. Among them, let us quote the problem of the vibrating string subject to solid friction or even the damped wave equation with a dry friction term. In the case of the vibrating 
string, Cabannes $[8,9]$ has obtained some partial results on finite time stabilization corresponding to particular initial data. However the general case of arbitrary initial data is still open.

The paper is organized as follows. In section 2 we derive the differential inclusion $(S)$ from its mechanical origin and we recall the result of finite time stabilization obtained in [1]. In sections 3 and 4 , we show that the geometry of the set $\partial \Phi(0)$ plays a central role in the asymptotic behaviour of $(S)$. Section 3 deals with the case of a smooth set $\partial \Phi(0)$. In this framework, we prove exponential decay results and we derive sufficient conditions ensuring finite time convergence. Section 4 is concerned with the asymptotic analysis of $(S)$ when the set $\partial \Phi(0)$ is a convex polyhedron.

\section{Preliminaries AND General Results}

Let us first describe the notations that we use throughout the paper. The linear space $\mathbb{R}^{n}$ is endowed with its canonical euclidean structure $\langle\cdot, \cdot\rangle$ and the corresponding norm $|$.$| . Let \mathbb{B}$ (resp. $\mathbb{S}$ ) be the unit euclidean ball of $\mathbb{R}^{n}$ (resp. the unit euclidean sphere) centered at 0 . Given a subset $A \subset \mathbb{R}^{n}$, we denote by $d(., A)$ the distance function to the set $A: d(x, A)=\inf _{y \in A}|x-y|$ for every $x \in \mathbb{R}^{n}$. Given another subset $B \subset \mathbb{R}^{n}$, we define the excess $e(A, B)$ of $A$ over $B$ by $e(A, B)=\sup _{x \in A} d(x, B)$. Throughout the paper, we also use the standard notations of convex analysis. Given a convex function $\Phi: \mathbb{R}^{n} \rightarrow \mathbb{R}$, the set $\partial \Phi(x)$ and the function $\Phi^{\prime}(x ;$.$) are respectively the subdifferential set at x \in \mathbb{R}^{n}$ and the directional derivative at $x$. The subdifferential of the absolute value function $||:. \mathbb{R} \rightarrow \mathbb{R}$ is the set-valued sign function, denoted by $\operatorname{sgn}: \operatorname{sgn}(x)=1$ if $x>0$, $\operatorname{sgn}(x)=-1$ if $x<0$ and $\operatorname{sgn}(0)=[-1,1]$. For a convex subset $C \subset \mathbb{R}^{n}$, we denote by $N_{C}$ the normal cone operator and by $\sigma_{C}$ the support function of $C$. We refer the reader to [19] for the definitions and basic properties relative to these notions.

2.1. Mechanical origin. The study of the vibrations of a string moving in a plane and subject to dry friction amounts to the determination of a function $u: \mathbb{R} \times \mathbb{R}_{+} \rightarrow$ $\mathbb{R}$ satisfying the following differential inclusion:

$$
\frac{\partial^{2} u}{\partial t^{2}}-\frac{\partial^{2} u}{\partial x^{2}} \in-a \operatorname{sgn}\left(\frac{\partial u}{\partial t}\right)-g\left(\frac{\partial u}{\partial t}\right),
$$

where $a>0, \operatorname{sgn}: \mathbb{R} \rightrightarrows \mathbb{R}$ is the set-valued sign function and $g: \mathbb{R} \rightarrow \mathbb{R}$ is a Lipschitz continuous function such that $r g(r) \geq 0$ for every $r \in \mathbb{R}$. The right member of (2.1) represents the friction term, which is the combination of a Coulomb friction and of another type of friction such as the one due the viscosity of a surrounding fluid. The reader is referred to $[7,12,17]$ for general features about the Coulomb model. By using a finite differencing scheme, the spatial discretization of (2.1) leads to

$$
\frac{d^{2} u_{i}}{d t^{2}}-\frac{u_{i+1}-2 u_{i}+u_{i-1}}{h^{2}} \in-a \operatorname{sgn}\left(\frac{d u_{i}}{d t}\right)-g\left(\frac{d u_{i}}{d t}\right),
$$

with $i=1,2, \ldots, n$ and $h=1 /(n+1)$. The previous inclusion can be rewritten as a vectorial problem by setting $U(t):=\left(u_{1}(t), \ldots, u_{n}(t)\right)^{T}$. For that purpose, let us define the function $\operatorname{Sgn}: \mathbb{R}^{n} \rightrightarrows \mathbb{R}^{n}$ by $\operatorname{Sgn}\left(u_{1}, \ldots, u_{n}\right):=\left(\operatorname{sgn}\left(u_{1}\right), \ldots, \operatorname{sgn}\left(u_{n}\right)\right)^{T}$ and the function $G: \mathbb{R}^{n} \rightarrow \mathbb{R}^{n}$ by $G\left(u_{1}, \ldots, u_{n}\right):=\left(g\left(u_{1}\right), \ldots, g\left(u_{n}\right)\right)^{T}$. We also 
define the symmetric positive definite matrix $A \in \mathcal{M}_{n}(\mathbb{R})$ by

$$
A:=\frac{1}{h^{2}}\left(\begin{array}{ccccc}
2 & -1 & 0 & \cdots & 0 \\
-1 & 2 & -1 & 0 & \vdots \\
0 & \ddots & \ddots & \ddots & 0 \\
\vdots & 0 & -1 & 2 & -1 \\
0 & \cdots & 0 & -1 & 2
\end{array}\right) .
$$

With these notations, inclusion (2.2) is equivalent to

$$
\ddot{U}(t)+a \operatorname{Sgn}(\dot{U}(t))+G(\dot{U}(t))+A U(t) \ni 0, \quad t \geq 0 .
$$

The set-valued function Sgn is the subdifferential of the norm function $x \mapsto \sum_{i=1}^{n}\left|x_{i}\right|$. If the function $g$ defined above is nondecreasing, it is the first derivative of a convex function. Under these conditions, the term $a \operatorname{Sgn}+G$ is the subdifferential of some convex function $\Phi: \mathbb{R}^{n} \rightarrow \mathbb{R}$. Due to the symmetry of the matrix $A$, the linear term $A U$ equals the gradient of the function $f: \mathbb{R}^{n} \rightarrow \mathbb{R}$ defined by $f:=\frac{1}{2}\langle A U, U\rangle$. These considerations lead us to rewrite inclusion (2.4) as

$$
\ddot{U}(t)+\partial \Phi(\dot{U}(t))+\nabla f(U(t)) \ni 0, \quad t \geq 0 .
$$

The modelling of friction by a subdifferential operator is classical in unilateral mechanics; see for instance $[2,18]$. The fact that the friction has a dry component is expressed by the condition $0 \in \operatorname{int}(\partial \Phi(0))$, as explained in [1]. For more details on the dynamics of a vibrating string subject to dry friction, the reader is referred to references $[5,8,9]$.

2.2. General results. Fundamental examples. Consider the functions $\Phi$ : $\mathbb{R}^{n} \rightarrow \mathbb{R}$ and $f: \mathbb{R}^{n} \rightarrow \mathbb{R}$ satisfying respectively the following assumptions:

$\left(\mathcal{H}_{\Phi}-i\right) \Phi$ is convex.

$\left(\mathcal{H}_{\Phi}-i i\right) 0 \in \operatorname{int}(\partial \Phi(0))$.

$\left(\mathcal{H}_{f}-i\right) f$ is of class $\mathcal{C}^{1}$ and $\nabla f$ is Lipschitz continuous on the bounded sets of $\mathbb{R}^{n}$.

$\left(\mathcal{H}_{f}-i i\right) f$ is bounded from below.

To be more concise, we denote by $(\mathcal{H})$ the set of hypotheses consisting of $\left(\mathcal{H}_{\Phi}-i, i i\right)$ and $\left(\mathcal{H}_{f}-i, i i\right)$. The mechanical considerations of the previous paragraph lead us to study the following second-order (in time) differential inclusion:

$$
\ddot{x}(t)+\partial \Phi(\dot{x}(t))+\nabla f(x(t)) \ni 0, \quad t \geq 0 .
$$

The next statement gathers the main general features about the dynamical system $(S)$. For every closed convex set $C \subset \mathbb{R}^{n}$, we denote by $C^{0}$ the unique element of minimal norm of $C$.

Theorem 2.1. Assume that hypotheses $(\mathcal{H})$ hold.

(i) For every $\left(x_{0}, \dot{x}_{0}\right) \in \mathbb{R}^{n} \times \mathbb{R}^{n}$, there exists a unique solution $x:\left[0,+\infty\left[\rightarrow \mathbb{R}^{n}\right.\right.$ of $(S)$ in the following sense:

(a) $x \in \mathcal{C}^{1}\left(\left[0,+\infty\left[, \mathbb{R}^{n}\right)\right.\right.$ and $\dot{x} \in \operatorname{Lip}\left([0, T], \mathbb{R}^{n}\right)$ for every $T>0$.

(b) $(S)$ is satisfied for almost every $t \in[0,+\infty[$.

(c) $x(0)=x_{0}$ and $\dot{x}(0)=\dot{x}_{0}$. 
(ii) For almost every $t \geq 0$, the following selection property holds:

$$
\ddot{x}(t)+[\partial \Phi(\dot{x}(t))+\nabla f(x(t))]^{0}=0 .
$$

(iii) $|\dot{x}| \in L^{1}\left(\left[0,+\infty[, \mathbb{R})\right.\right.$ and $\lim _{t \rightarrow+\infty} \dot{x}(t)=0$.

(iv) Denoting by $\Gamma \subset \mathbb{R}_{+}$the subset on which the map $\dot{x}$ is derivable and the inclusion $(S)$ is satisfied, we have $\lim _{t \rightarrow+\infty, t \in \Gamma} \ddot{x}(t)=0$.

(v) $\lim _{t \rightarrow+\infty} x(t)=x_{\infty}$ for some $x_{\infty} \in \mathbb{R}^{n}$ satisfying $-\nabla f\left(x_{\infty}\right) \in \partial \Phi(0)$.

Proof. We refer the reader to [1, Theorem 2.1] for a detailed proof of $(i)$. Item (iii) is proved in [1, Propositions 3.1 and 3.2]. Assertion $(v)$ results from [1, Theorem 3.1]. Let us now prove $(i i)$. Remark that the dynamical system $(S)$ can be rewritten as a first-order in time inclusion. Indeed, by setting $y(t)=\dot{x}(t)$ and $g(t)=-\nabla f(x(t))$, the system $(S)$ can be equivalently rewritten as the following nonautonomous inclusion:

$$
\dot{y}(t)+\partial \Phi(y(t)) \ni g(t) .
$$

Since the function $g$ is Lipschitz continuous on $\mathbb{R}_{+}$, hence absolutely continuous, it suffices to apply $[6$, Proposition 3.3$]$ to find the selection property $(i i)$. Let us finally prove $(i v)$. Let $M>0$ be a Lipschitz constant of the map $\nabla f$ over the bounded set $\{x(t), t \geq 0\}$; the constant $M$ is finite in view of $\left(\mathcal{H}_{f}-i\right)$. It is immediate that we check that $|\dot{g}(t)| \leq M|\dot{x}(t)|$ for almost every $t \geq 0$. In view of (iii), it ensues that $\dot{g} \in L^{1}\left(\left[0,+\infty\left[, \mathbb{R}^{n}\right)\right.\right.$. By applying [6, Theorem 3.10] to the system $(2.5)$, we immediately deduce that $\lim _{t \rightarrow+\infty, t \in \Gamma} \dot{y}(t)=0$, whence $(i v)$.

Given a subset $C \subset \mathbb{R}^{n}$, it is immediate to check that the function $\Phi:=\sigma_{C}$ satisfies $\left(\mathcal{H}_{\Phi}-i, i i\right)$ if and only if the set $C$ is closed, convex, bounded and such that $0 \in \operatorname{int}(C)$. This remark gives rise to the following examples, obtained by particularizing the set $C$.

Example 2.2. Let $L \in M_{n}(\mathbb{R})$ be a symmetric positive definite matrix. Define the set $C:=\mathbb{B}_{L}=\left\{x \in \mathbb{R}^{n}, \quad\langle L x, x\rangle \leq 1\right\}$ and the associated norm $|\cdot|_{L}$ given by $|x|_{L}=\sqrt{\langle L x, x\rangle}$. It is easy to check that, for every $x \in \mathbb{R}^{n}, \sigma_{\mathbb{B}_{L}}(x)=\sqrt{\left\langle L^{-1} x, x\right\rangle}=$ $|x|_{L^{-1}}$. An immediate computation shows that $\sigma_{\mathbb{B}_{L}}$ is differentiable on $\mathbb{R}^{n} \backslash\{0\}$ and that:

$$
\forall x \in \mathbb{R}^{n} \backslash\{0\}, \quad \nabla \sigma_{\mathbb{B}_{L}}(x)=\frac{L^{-1} x}{\sqrt{\left\langle L^{-1} x, x\right\rangle}} .
$$

The subdifferential set of $\sigma_{\mathbb{B}_{L}}$ at 0 equals $\partial \sigma_{\mathbb{B}_{L}}(0)=\mathbb{B}_{L}$.

Example 2.3. Let $C:=\mathbb{B}_{p}=\left\{x \in \mathbb{R}^{n}, \quad \sum_{i=1}^{n}\left|x_{i}\right|^{p} \leq 1\right\}$ for some $\left.p \in\right] 1, \infty[$. Let $q$ be the conjugate exponent of $p$ defined by $1 / p+1 / q=1$. It is well-known that for all $x \in \mathbb{R}^{n}, \sigma_{C}(x)=|x|_{q}=\left(\sum_{i=1}^{n}\left|x_{i}\right|^{q}\right)^{1 / q}$. An easy computation shows that $\partial|\cdot|_{q}$ is given by:

$$
\partial|\cdot|_{q}(x)=\left\{\begin{array}{cl}
\frac{1}{|x|_{q}^{q / p}}\left(\left|x_{1}\right|^{q-1} \operatorname{sgn}\left(x_{1}\right), \ldots,\left|x_{n}\right|^{q-1} \operatorname{sgn}\left(x_{n}\right)\right) & \text { if } x \neq 0 \\
\mathbb{B}_{p} & \text { if } x=0 .
\end{array}\right.
$$

Example 2.4. Let $C:=\mathbb{B}_{\infty}=\left\{x \in \mathbb{R}^{n}, \quad \max _{i=1}^{n}\left|x_{i}\right| \leq 1\right\}$. We classically have for all $x \in \mathbb{R}^{n}, \sigma_{C}(x)=|x|_{1}=\sum_{i=1}^{n}\left|x_{i}\right|$, and it is then immediate that $\partial|\cdot|_{1}(x)=\left(\operatorname{sgn}\left(x_{1}\right), \ldots, \operatorname{sgn}\left(x_{n}\right)\right)$. In particular, we have $\partial|\cdot|_{1}(0)=\mathbb{B}_{\infty}$. 
Example 2.5. Let $C:=\mathbb{B}_{1}=\left\{x \in \mathbb{R}^{n}, \quad \sum_{i=1}^{n}\left|x_{i}\right| \leq 1\right\}$. It is classical that for all $x \in \mathbb{R}^{n}, \sigma_{C}(x)=|x|_{\infty}=\max _{i=1}^{n}\left|x_{i}\right|$ and since $|\cdot|_{\infty}$ is the supremum of a finite collection of convex functions, the usual rule of subdifferentiation yields:

$$
\partial|\cdot|_{\infty}(x)=\left\{\begin{array}{cc}
\operatorname{conv}\left\{\operatorname{sgn}\left(x_{i}\right) e_{i}, i \in I_{x}\right\} & \text { if } x \neq 0 \\
\mathbb{B}_{1} & \text { if } \quad x=0
\end{array}\right.
$$

where $\left(e_{1}, \ldots, e_{n}\right)$ is the canonical basis of $\mathbb{R}^{n}$ and $I_{x}=\left\{i \in\{1, \ldots, n\},\left|x_{i}\right|=|x|_{\infty}\right\}$ is the set of active indices.

The previous examples will serve us as a guideline throughout the paper.

2.3. Finite time stabilization and counterexamples. The most remarkable feature of the dry friction condition $0 \in \operatorname{int}(\partial \Phi(0))$ is the finite time stabilization of the associate dynamics. A first result in this direction is given by the following statement, where the limit $x_{\infty}$ is assumed to satisfy some interior condition.

Proposition 2.6. Under hypotheses $(\mathcal{H})$, let $x$ be the unique solution of the differential inclusion $(S)$ and let us denote by $x_{\infty}:=\lim _{t \rightarrow+\infty} x(t)$ its limit when $t \rightarrow+\infty$. If $-\nabla f\left(x_{\infty}\right) \in \operatorname{int}(\partial \Phi(0))$, then there exists $t_{0} \geq 0$ such that $x(t)=x_{\infty}$ for every $t \geq t_{0}$.

The reader is referred to [1, Theorem 3.2] for the proof of this result. When condition $-\nabla f\left(x_{\infty}\right) \in \operatorname{int}(\partial \Phi(0))$ does not hold, the trajectory $x$ may not converge in a finite time, as shown by the following examples.

Example 2.7. Take $n=1, \Phi:=|\cdot|+|.|^{2}$ (so that $\left.\partial \Phi(0)=[-1,1]\right)$ and $f:=|\cdot|^{2} / 2$. The differential inclusion $(S)$ then reduces to

$$
\ddot{x}(t)+\operatorname{sgn}(\dot{x}(t))+2 \dot{x}(t)+x(t) \ni 0 .
$$

Let us choose as initial conditions $x(0)=2$ and $\dot{x}(0)=-1$. We let the reader check that the unique solution to $(S)$ is given by $x(t)=1+e^{-t}, t \geq 0$. The trajectory tends toward the value $x_{\infty}=1$, which satisfies $-f^{\prime}\left(x_{\infty}\right)=-1 \in \operatorname{bd}(\partial \Phi(0))$. However the convergence does not hold in a finite time.

Example 2.8. Take $n=1, \Phi:=|$.$| and f:=-|\cdot|^{2} / 2$. The underlying differential inclusion is:

$$
\ddot{x}(t)+\operatorname{sgn}(\dot{x}(t))-x(t) \ni 0 .
$$

We let the reader check that the unique solution to $(S)$ satisfying $(x(0), \dot{x}(0))=$ $(0,1)$ is given by $x(t)=1-e^{-t}, t \geq 0$. The trajectory $x$ exponentially tends toward $x_{\infty}=1$.

In the previous two examples, the rate of convergence toward $x_{\infty}$ is exponential. We now give an example in which the convergence is polynomial.

Example 2.9. Take $n=1, \Phi:=||+.2 / 3|\cdot|^{3 / 2}+4 / 5|\cdot|^{5 / 2}$ and $f:=|\cdot|^{2} / 2$. This gives rise to the following differential inclusion:

$$
\ddot{x}(t)+\operatorname{sgn}(\dot{x}(t))\left(1+|\dot{x}(t)|^{1 / 2}+2|\dot{x}(t)|^{3 / 2}\right)+x(t) \ni 0 .
$$

Consider the inclusion $(S)$ on $[1,+\infty[$ and take as initial conditions $(x(1), \dot{x}(1))=$ $(2,-1)$. It is immediate to check that the unique solution to the Cauchy problem is equal to $x(t)=1+1 / t, t \geq 1$. 
The purpose of the paper is to study the asymptotic properties of $(S)$ when the vector $-\nabla f\left(x_{\infty}\right)$ belongs to the boundary of the set $\partial \Phi(0)$. We will show that, in a rather large setting the convergence rate is (at least) exponential. We will also determine sufficient conditions on $\Phi$ and $f$ ensuring the finite time stabilization of $(S)$.

2.4. Structure of the zeroes of the map $\dot{x}$. Let us now study the topological structure of the set $\mathcal{D}=\left\{t \in \mathbb{R}_{+}, \quad|\dot{x}(t)|=0\right\}$.

Proposition 2.10. Under the hypotheses $(\mathcal{H})$, let $x$ be the unique solution to the dynamical system $(S)$ and let us set $\mathcal{D}:=\left\{t \in \mathbb{R}_{+}, \quad|\dot{x}(t)|=0\right\}$. Then, either the set $\mathcal{D}$ equals the interval $\left[t_{0},+\infty\left[\right.\right.$ for some $t_{0} \geq 0$ or the set $\mathcal{D}$ is discrete and countable (hence of zero measure).

Proof. Assume that $\mathcal{D}$ is not equal to any interval $\left[t_{0},+\infty\left[\right.\right.$ with $t_{0} \geq 0$. Consider any $t_{1}>0$ satisfying $\left|\dot{x}\left(t_{1}\right)\right|=0$ (if such an element does not exist, the conclusion is trivial) and let us prove that it is an isolated point of $\mathcal{D}$. Let us first remark that we necessarily have $-\nabla f\left(x\left(t_{1}\right)\right) \notin \partial \Phi(0)$. Indeed, if $-\nabla f\left(x\left(t_{1}\right)\right) \in \partial \Phi(0)$, then the constant function equal to $x\left(t_{1}\right)$ on $\left[t_{1},+\infty\right.$ [ is a solution to $(S)$, and from the uniqueness result we derive that $x(t)=x\left(t_{1}\right)$ for every $t \geq t_{1}$, a contradiction.

Since $-\nabla f\left(x\left(t_{1}\right)\right) \notin \partial \Phi(0)$, it is possible to strictly separate the convex compact set $\{0\}$ from the closed convex set $\partial \Phi(0)+\nabla f\left(x\left(t_{1}\right)\right)$. More precisely, there exist $p \in \mathbb{R}^{n}$ and $m \in \mathbb{R}_{+}^{*}$ such that:

$$
\forall \xi \in \partial \Phi(0), \quad\left\langle\xi+\nabla f\left(x\left(t_{1}\right)\right), p\right\rangle>m .
$$

By using the graph-closedness property of the operator $\partial \Phi$ and the continuity of $\nabla f$, one can easily show that there exists $\varepsilon>0$ such that

$$
\forall t \in] t_{1}-\varepsilon, t_{1}+\varepsilon[, \quad \forall \xi \in \partial \Phi(\dot{x}(t)), \quad\langle\xi+\nabla f(x(t)), p\rangle>m .
$$

In view of $(S)$, we deduce that, for almost every $t \in] t_{1}-\varepsilon, t_{1}+\varepsilon[,\langle\ddot{x}(t), p\rangle<-m$. Let us integrate this inequality on $\left[t_{1}, t\right]$ to obtain:

$$
\forall t \in] t_{1}-\varepsilon, t_{1}+\varepsilon\left[, \quad|\langle\dot{x}(t), p\rangle|>m\left|t-t_{1}\right| .\right.
$$

Therefore, we have $|\dot{x}(t)| \neq 0$ for every $t \in] t_{1}-\varepsilon, t_{1}+\varepsilon[$ and hence $\mathcal{D} \cap] t_{1}-\varepsilon, t_{1}+\varepsilon[=$ $\left\{t_{1}\right\}$, i.e. $t_{1}$ is isolated in $\mathcal{D}$. Since this is true for every $t_{1} \in \mathcal{D}$, the set $\mathcal{D}$ is discrete. On the other hand, the set $\mathcal{D}$ is clearly closed in view of the continuity of the map $t \mapsto|\dot{x}(t)|$. We infer that every bounded subset of $\mathcal{D}$ is finite. We conclude that the set $\mathcal{D}$ is countable as a countable union of finite sets.

\section{Analysis of the boundary Case (I): $\partial \Phi(0)$ SMooth}

Let us recall that a convex set $C \subset \mathbb{R}^{n}$ is said to be smooth at a point $\bar{x} \in C$ if $N_{C}(\bar{x})$ is reduced to a half-line. The set $C$ is said to be smooth if it is smooth at every point of its boundary.

3.1. Analysis of the mode of convergence. The next result shows that if the set $\partial \Phi(0)$ is smooth, then either the solution $x$ to $(S)$ converges in a finite time or the direction of the velocity vector $\dot{x}(t)$ tends to be normal to the boundary of $\partial \Phi(0)$ when $t \rightarrow+\infty$. Let us recall that $\Gamma$ is the subset of $\mathbb{R}_{+}$on which the map $\dot{x}$ is derivable and the inclusion $(S)$ is satisfied. On the other hand, we denote by $\mathcal{D}$ the set of the zeroes of the map $t \mapsto|\dot{x}(t)|$. 
Proposition 3.1. Under hypotheses $(\mathcal{H})$, let $x$ be the unique solution of the differential inclusion $(S)$ and let us denote by $x_{\infty}:=\lim _{t \rightarrow+\infty} x(t)$ its limit when $t \rightarrow+\infty$. Assume that the set $\partial \Phi(0)$ is smooth at the point $-\nabla f\left(x_{\infty}\right)$ and consider the unique vector $u \in \mathbb{R}^{n}$ defined by $|u|=1$ and $N_{\partial \Phi(0)}\left(-\nabla f\left(x_{\infty}\right)\right)=\mathbb{R}_{+} u$. Then, either the solution $x$ converges in a finite time or we have

$$
\begin{gathered}
\lim _{t \rightarrow+\infty, t \notin \mathcal{D}} \frac{\dot{x}(t)}{|\dot{x}(t)|}=u, \\
x(t)=x_{\infty}-\left(\int_{t}^{+\infty}|\dot{x}(s)| d s\right) u+o\left(\int_{t}^{+\infty}|\dot{x}(s)| d s\right) .
\end{gathered}
$$

As a consequence, we have the equivalence $\left|x(t)-x_{\infty}\right| \sim \int_{t}^{+\infty}|\dot{x}(s)| d s$ when $t \rightarrow$ $+\infty$ and we deduce that either $x(t)=x_{\infty}$ for $t$ large enough or $\left|x(t)-x_{\infty}\right|>0$ for $t$ large enough.

Proof. Assume that the trajectory $x$ does not converge in a finite time. We deduce from Proposition 2.10 that the set $\mathcal{D}$ is discrete. Let $d \in \mathbb{R}^{n}$ be a limit point of $\left\{\dot{x}(t) /|\dot{x}(t)|, \quad t \in \mathbb{R}_{+} \backslash \mathcal{D}\right\}:$ there exists a sequence $\left(t_{n}\right) \subset \mathbb{R}_{+} \backslash \mathcal{D}$ tending to $+\infty$ such that $\lim _{n \rightarrow+\infty} \dot{x}\left(t_{n}\right) /\left|\dot{x}\left(t_{n}\right)\right|=d$. From the continuity of the map $\dot{x}$, we can assume without loss of generality that $\left(t_{n}\right) \subset \Gamma \backslash \mathcal{D}$ and hence

$$
-\ddot{x}\left(t_{n}\right)-\nabla f\left(x\left(t_{n}\right)\right) \in \partial \Phi\left(\dot{x}\left(t_{n}\right)\right) .
$$

Let $\xi \in \mathbb{R}^{n}$ be an element of $\partial \Phi(0)$; from the monotonicity of $\partial \Phi$, we have

$$
\left\langle-\ddot{x}\left(t_{n}\right)-\nabla f\left(x\left(t_{n}\right)\right)-\xi, \frac{\dot{x}\left(t_{n}\right)}{\left|\dot{x}\left(t_{n}\right)\right|}\right\rangle \geq 0 .
$$

Take the limit in the previous inequality when $n \rightarrow+\infty$. In view of Theorem 2.1 (iv), we have $\lim _{n \rightarrow+\infty} \ddot{x}\left(t_{n}\right)=0$ so that we find $\left\langle\nabla f\left(x_{\infty}\right)+\xi, d\right\rangle \leq 0$. Since the last inequality holds true for every $\xi \in \partial \Phi(0)$, we deduce that $d \in N_{\partial \Phi(0)}\left(-\nabla f\left(x_{\infty}\right)\right)=$ $\mathbb{R}_{+} u$. On the other hand, we have $\dot{x}(t) /|\dot{x}(t)| \in \mathbb{S}$ and we infer that $d \in \mathbb{S} \cap \mathbb{R}_{+} u=$ $\{u\}$. As a consequence, the vector $u$ is the unique limit point of the bounded map $t \mapsto \dot{x}(t) /|\dot{x}(t)|$, and we conclude that equality (3.1) holds. Notice that this equality can be equivalently rewritten as $\dot{x}(t)=|\dot{x}(t)| u+o(|\dot{x}(t)|)$ when $t \rightarrow+\infty$. Integrating the last equality on $[t,+\infty[$ and taking into account the fact that $|\dot{x}| \in$ $L^{1}(0,+\infty)$, we obtain (3.2). The other assertions are immediate consequences of $(3.2)$.

3.2. Estimation of the convergence rate. In the sequel of the paper, the convexity (or even the strong convexity) of $f$ will play a crucial role in the asymptotic analysis of $(S)$, as suggested by the examples of section 2.3. Let us recall that the smooth function $f: \mathbb{R}^{n} \rightarrow \mathbb{R}$ is said to be strongly convex if there exists $M>0$ such that

$$
\forall x, y \in \mathbb{R}^{n}, \quad\langle\nabla f(x)-\nabla f(y), x-y\rangle \geq M|x-y|^{2} .
$$

It is easy to show that the previous formulation is equivalent to the following one:

$$
\forall x, y \in \mathbb{R}^{n}, \quad f(x) \geq f(y)+\langle\nabla f(y), x-y\rangle+M / 2|x-y|^{2} .
$$

The next result shows that either the solutions to $(S)$ converge in a finite time or the convergence rate is exponential. 
Theorem 3.2. Under hypotheses $(\mathcal{H})$, suppose that there exist $\eta>0$ and $\alpha \geq 0$ such that

$$
|x| \leq \eta \quad \Longrightarrow \quad e(\partial \Phi(x), \partial \Phi(0)) \leq \alpha|x| .
$$

Assume moreover that the function $f$ satisfies the strong convexity assumption (3.3). Let $x$ be the unique solution of the differential inclusion $(S)$, and let us denote by $x_{\infty}:=\lim _{t \rightarrow+\infty} x(t)$ its limit when $t \rightarrow+\infty$. If the set $\partial \Phi(0)$ is smooth at the point $-\nabla f\left(x_{\infty}\right)$, then one of the following cases holds:

(i) There exists $t_{0} \geq 0$ such that $x(t)=x_{\infty}$ for every $t \geq t_{0}$.

(ii) There exist $t_{1} \geq 0, A, B>0$ and $\gamma_{1}, \gamma_{2}>0$ such that for every $t \geq t_{1}$,

$$
\left|x(t)-x_{\infty}\right| \geq A e^{-\gamma_{1} t} \quad \text { and } \quad \int_{t}^{+\infty}\left|x(s)-x_{\infty}\right| d s \leq B e^{-\gamma_{2} t} .
$$

Any positive exponent $\gamma_{1}>\alpha$ (resp. $\gamma_{2}<M / \alpha$ ) satisfies the previous estimate. If moreover $\alpha<\sqrt{M}$, then case $(i)$ necessarily occurs.

Proof. Let us assume that case $(i)$ does not hold, i.e. the solution $x$ does not converge toward $x_{\infty}$ in a finite time. For every $t \in \Gamma$, we have $-\ddot{x}(t)-\nabla f(x(t)) \in$ $\partial \Phi(\dot{x}(t))$. Let us define $\xi(t)$ as the unique element of $\partial \Phi(0)$ such that

$$
d(-\ddot{x}(t)-\nabla f(x(t)), \partial \Phi(0))=|\xi(t)+\ddot{x}(t)+\nabla f(x(t))| .
$$

Since the set $\partial \Phi(0)$ is smooth at the point $-\nabla f\left(x_{\infty}\right)$, there exists $u \in \mathbb{R}^{n}$ such that $|u|=1$ and $N_{\partial \Phi(0)}\left(-\nabla f\left(x_{\infty}\right)\right)=\mathbb{R}_{+} u$. Let us write that

$$
\begin{aligned}
\langle\ddot{x}(t), u\rangle=\langle\xi(t)+\ddot{x}(t)+\nabla f(x(t)), u\rangle-\langle\xi(t) & \left.+\nabla f\left(x_{\infty}\right), u\right\rangle \\
& +\left\langle\nabla f(x(t))-\nabla f\left(x_{\infty}\right),-u\right\rangle,
\end{aligned}
$$

and let us evaluate each term of the right member. From the definition of $\xi(t)$ we have for every $t \in \Gamma$ :

$$
|\xi(t)+\ddot{x}(t)+\nabla f(x(t))| \leq \sup _{y \in \partial \Phi(\dot{x}(t))} d(y, \partial \Phi(0))=e(\partial \Phi(\dot{x}(t)), \partial \Phi(0)) .
$$

Since $\lim _{t \rightarrow+\infty} \dot{x}(t)=0$, there exists $t_{0} \geq 0$ such that $|\dot{x}(t)| \leq \eta$ for every $t \geq t_{0}$. Hence we deduce from assumption (3.4) and the previous inequality that

$$
\forall t \in\left[t_{0},+\infty[\cap \Gamma, \quad|\xi(t)+\ddot{x}(t)+\nabla f(x(t))| \leq \alpha|\dot{x}(t)| .\right.
$$

On the other hand, since $u \in N_{\partial \Phi(0)}\left(-\nabla f\left(x_{\infty}\right)\right)$ and $\xi(t) \in \partial \Phi(0)$, we infer

$$
\left\langle u, \xi(t)+\nabla f\left(x_{\infty}\right)\right\rangle \leq 0 .
$$

From Proposition 3.1, we have $x(t)-x_{\infty}=-\left|x(t)-x_{\infty}\right| u+o\left(\left|x(t)-x_{\infty}\right|\right)$ and $\left|x(t)-x_{\infty}\right|>0$ for $t$ large enough. We deduce that

$$
\left\langle\nabla f(x(t))-\nabla f\left(x_{\infty}\right),-u\right\rangle=\left\langle\nabla f(x(t))-\nabla f\left(x_{\infty}\right), \frac{x(t)-x_{\infty}}{\left|x(t)-x_{\infty}\right|}+o(1)\right\rangle .
$$

Since $\nabla f$ is Lipschitz continuous in the neighborhood of $x_{\infty}$, we have

$$
\left\langle\nabla f(x(t))-\nabla f\left(x_{\infty}\right), o(1)\right\rangle=o\left(\left|x(t)-x_{\infty}\right|\right) \quad \text { when } \quad t \rightarrow+\infty .
$$

Hence, in view of (3.3) and (3.9), we infer that

$$
\left\langle\nabla f(x(t))-\nabla f\left(x_{\infty}\right),-u\right\rangle \geq M\left|x(t)-x_{\infty}\right|+o\left(\left|x(t)-x_{\infty}\right|\right) .
$$


Let us set $F(t):=\int_{t}^{+\infty}|\dot{x}(s)| d s$; from Proposition 3.1, we have the equivalence $\left|x(t)-x_{\infty}\right| \sim F(t)$ when $t \rightarrow+\infty$. As a consequence, the previous inequality can be rewritten as:

$$
\left\langle\nabla f(x(t))-\nabla f\left(x_{\infty}\right),-u\right\rangle \geq M F(t)+o(F(t)) .
$$

In view of (3.6), we deduce from (3.7), (3.8) and (3.10) that

$$
\langle\ddot{x}(t), u\rangle \geq-\alpha|\dot{x}(t)|+M F(t)+o(F(t)) .
$$

It ensues that $\int_{0}^{+\infty} F(s) d s<+\infty$ so that we can define the function $G: \mathbb{R}_{+} \rightarrow \mathbb{R}$ by $G(t)=\int_{t}^{+\infty} F(s) d s$. Now integrate inequality (3.11) on $[t,+\infty[$ to find:

$$
-\langle\dot{x}(t), u\rangle \geq-\alpha F(t)+M G(t)+o(G(t)) .
$$

From equality (3.1) of Proposition 3.1, we infer that

$$
|\dot{x}(t)|+o(|\dot{x}(t)|)+M G(t)+o(G(t)) \leq \alpha F(t) .
$$

As a consequence, for every $\varepsilon \in] 0,1\left[\right.$, there exist $t_{1} \geq t_{0}$ such that for every $t \geq t_{1}$

$$
(1-\varepsilon)|\dot{x}(t)| \leq \alpha F(t) \quad \text { and } \quad(1-\varepsilon) M G(t) \leq \alpha F(t) .
$$

If $\alpha=0$, we deduce from the first inequality that $|\dot{x}(t)|=0$ for every $t \geq t_{1}$. Now assume that $\alpha>0$. Setting $\gamma_{1}=\alpha /(1-\varepsilon)$ and $\gamma_{2}=(1-\varepsilon) M / \alpha$, the previous two inequalities can be rewritten as

$$
\dot{F}(t)+\gamma_{1} F(t) \geq 0 \quad \text { and } \quad \dot{G}(t)+\gamma_{2} G(t) \leq 0 .
$$

An elementary integration of these inequalities on $\left[t_{1}, t\right]$ yields respectively:

$$
F(t) \geq F\left(t_{1}\right) e^{-\gamma_{1}\left(t-t_{1}\right)} \quad \text { and } \quad G(t) \leq G\left(t_{1}\right) e^{-\gamma_{2}\left(t-t_{1}\right)} .
$$

The conclusion follows from the equivalence $\left|x(t)-x_{\infty}\right| \sim \int_{t}^{+\infty}|\dot{x}(s)| d s$ when $t \rightarrow+\infty$.

Let us now prove the last assertion of the theorem. Let us argue by contradiction and assume that case $(i i)$ holds. An immediate integration of the first inequality of (3.5) on $\left[t,+\infty\left[\right.\right.$ shows that $\int_{t}^{+\infty}\left|x(s)-x_{\infty}\right| d s \geq A / \gamma_{1} e^{-\gamma_{1} t}$, for every $t \geq t_{1}$. In view of the second inequality of (3.5), the exponents must satisfy $\gamma_{2} \leq \gamma_{1}$. Since this is true for every $\gamma_{1}>\alpha$ and $\gamma_{2}<M / \alpha$, we conclude that $M \leq \alpha^{2}$, which contradicts the assumption.

Let us now briefly comment on the assumptions of Theorem 3.2. If condition (3.4) is not fulfilled, then the convergence rate may not be exponential; see for instance Example 2.9 where it is polynomial. In the same direction, Example 2.8 shows that without convexity assumption on $f$, the trajectory $x$ may not converge in a finite time, even if $\alpha=0$. In the next corollary, we focus on the particular case where $\Phi:=\sigma_{C}+\Psi$ for some convex set $C \subset \mathbb{R}^{n}$ and some smooth convex function $\Psi: \mathbb{R}^{n} \rightarrow \mathbb{R}$.

Corollary 3.3. Let $C$ be a closed convex subset of $\mathbb{R}^{n}$ which is bounded and such that $0 \in \operatorname{int}(C)$. Consider a convex function $\Psi: \mathbb{R}^{n} \rightarrow \mathbb{R}$ of class $\mathcal{C}^{1}$ such that there exist $\eta>0$ and $\alpha \geq 0$ satisfying

$$
|x| \leq \eta \quad \Longrightarrow \quad|\nabla \Psi(x)| \leq \alpha|x|
$$

Assume that the function $\Phi: \mathbb{R}^{n} \rightarrow \mathbb{R}$ is defined by $\Phi:=\sigma_{C}+\Psi$. Suppose moreover that the function $f: \mathbb{R}^{n} \rightarrow \mathbb{R}$ verifies $\left(\mathcal{H}_{f}-i, i i\right)$ and the strong convexity 
assumption (3.3). If the set $C$ is smooth at the point $-\nabla f\left(x_{\infty}\right)$, then the solution $x$ of $(S)$ satisfies the same conclusions as in Theorem 3.2 .

Proof. Let us compute the excess $e(\partial \Phi(x), \partial \Phi(0))$. It is immediate to check that $\partial \sigma_{C}(0)=C$ and $\partial \sigma_{C}(x) \subset C$ for every $x \in \mathbb{R}^{n}$. Hence,

$$
\begin{aligned}
e(\partial \Phi(x), \partial \Phi(0)) & \leq e(\nabla \Psi(x)+C, C)=\sup _{y \in C} d(\nabla \Psi(x)+y, C) \\
& \leq \sup _{y \in C}|\nabla \Psi(x)+y-y|=|\nabla \Psi(x)| \leq \alpha|x| .
\end{aligned}
$$

It now suffices to apply Theorem 3.2.

If the set $C$ is smooth at every point of its boundary, the conclusions of the previous corollary automatically hold without condition on $x_{\infty}$. This is for example the case when $C=\mathbb{B}_{L}$ (see Example 2.2) or $C=\mathbb{B}_{p}$ for some $\left.p \in\right] 1,+\infty[$ (see Example 2.3).

Let us end this section by a result of finite time stabilization. We assume that the friction term can be decomposed as the sum of an isotropic dry friction and a viscous one. If the viscous component is small enough, the oscillator stabilizes in a finite time.

Corollary 3.4. Given $a>0, b \geq 0$, assume that $\Phi:=a|\cdot|+b|\cdot|^{2} / 2$. Suppose moreover that the function $f$ satisfies $\left(\mathcal{H}_{f}-i, i i\right)$ and that there exists $M>0$ such that (3.3) is fulfilled. If $b \in[0, \sqrt{M}[$, every solution to $(S)$ stabilizes in a finite time.

Proof. It suffices to apply Corollary 3.3 with $C:=a \mathbb{B}$ and $\Psi:=b|.|^{2} / 2$.

Assume now that the function $f$ is of class $\mathcal{C}^{2}$ and let us denote by $\lambda_{1}\left(\nabla^{2} f(x)\right)$ the smallest eigenvalue of the matrix $\nabla^{2} f(x)$. If the quantity $\inf _{x \in \mathbb{R}^{n}} \lambda_{1}\left(\nabla^{2} f(x)\right)$ is positive, then a simple application of Corollary 3.4 yields:

$$
b \in\left[0, \inf _{x \in \mathbb{R}^{n}} \sqrt{\lambda_{1}\left(\nabla^{2} f(x)\right)}[\Longrightarrow \text { every solution to }(S) \text { stabilizes in a finite time. }\right.
$$

This last result presents striking similarities with the corresponding one of DíazMillot [11, Theorem 2].

\section{Analysis of the Boundary CASE (II): $\partial \Phi(0)$ CONVEX POlyHEdRon}

We now focus our attention on the case where the set $\partial \Phi(0)$ is a convex polyhedron. The key property that will be exploited next is the following one: let $C \subset \mathbb{R}^{n}$ be a convex polyhedron and $\bar{x} \in C$. Then, there exists a neighbourhood $V$ of $\bar{x}$ such that

$$
\forall x \in V, \quad N_{C}(x) \subset N_{C}(\bar{x}) .
$$

This property will play a crucial role in the asymptotic analysis of $(S)$.

4.1. Analysis of the mode of convergence. Denoting by $x$ the unique solution to $(S)$, the next result shows that the velocity vector $\dot{x}(t)$ is normal to the boundary of $\partial \Phi(0)$ for $t$ large enough. We need the following technical assumption:

$$
\lim _{x \rightarrow 0} e\left(\partial \Phi(x), \partial\left[\Phi^{\prime}(0 ; .)\right](x)\right)=0,
$$


where $e(A, B)$ denotes the excess of the set $A$ over the set $B$. It is immediate to check that assumption (4.2) holds when $\Phi=\sigma_{C}+\Psi$, for some convex set $C \subset \mathbb{R}^{n}$ and some convex function $\Psi: \mathbb{R}^{n} \rightarrow \mathbb{R}$ such that $\partial \Psi(0)=\{0\}$.

Proposition 4.1. Under the hypotheses $(\mathcal{H})$, assume that $\partial \Phi(0)$ is a convex polyhedron and that (4.2) holds. Let $x$ be the unique solution to the dynamical system $(S)$ and let us set $x_{\infty}:=\lim _{t \rightarrow+\infty} x(t)$. Then there exists $t_{*} \geq 0$ such that

$$
\forall t \geq t_{*}, \quad \dot{x}(t) \in N_{\partial \Phi(0)}\left(-\nabla f\left(x_{\infty}\right)\right) .
$$

Proof. For every $t \in \Gamma$, we have: $-\ddot{x}(t)-\nabla f(x(t)) \in \partial \Phi(\dot{x}(t))$. Let us define $\xi(t)$ as the unique element of $\partial\left[\Phi^{\prime}(0 ;).\right](\dot{x}(t))$ such that

$$
d\left(-\ddot{x}(t)-\nabla f(x(t)), \partial\left[\Phi^{\prime}(0 ; .)\right](\dot{x}(t))\right)=|\xi(t)+\ddot{x}(t)+\nabla f(x(t))| .
$$

From the definition of $\xi(t)$ we have for every $t \in \Gamma$ :

$$
\begin{aligned}
|\xi(t)+\ddot{x}(t)+\nabla f(x(t))| & \leq \sup _{y \in \partial \Phi(\dot{x}(t))} d\left(y, \partial\left[\Phi^{\prime}(0 ; .)\right](\dot{x}(t))\right) \\
& =e\left(\partial \Phi(\dot{x}(t)), \partial\left[\Phi^{\prime}(0 ; .)\right](\dot{x}(t))\right) .
\end{aligned}
$$

Since $\lim _{x \rightarrow 0} e\left(\partial \Phi(x), \partial\left[\Phi^{\prime}(0 ;).\right](x)\right)=0$ and $\lim _{t \rightarrow+\infty} \dot{x}(t)=0$, we deduce from the previous inequality that $\lim _{t \rightarrow+\infty, t \in \Gamma} \xi(t)+\ddot{x}(t)+\nabla f(x(t))=0$. Recalling that $\lim _{t \rightarrow+\infty, t \in \Gamma} \ddot{x}(t)=0$ (cf. Theorem $\left.2.1(i v)\right)$ and that $\lim _{t \rightarrow+\infty} \nabla f(x(t))=$ $\nabla f\left(x_{\infty}\right)$, we infer that $\lim _{t \rightarrow+\infty, t \in \Gamma} \xi(t)=-\nabla f\left(x_{\infty}\right)$. Let us apply property (4.1) with $C:=\partial \Phi(0)$ and $\bar{x}:=-\nabla f\left(x_{\infty}\right) \in \partial \Phi(0)$. Since $\lim _{t \rightarrow+\infty, t \in \Gamma} \xi(t)=$ $-\nabla f\left(x_{\infty}\right)$, there exists $t_{*} \geq 0$ such that $\xi(t) \in V$ for almost every $t \geq t_{*}$. Recalling that $\partial\left[\Phi^{\prime}(0 ;).\right]=\left(N_{\partial \Phi(0)}\right)^{-1}$, it ensues that

$$
\dot{x}(t) \in N_{\partial \Phi(0)}(\xi(t)) \subset N_{\partial \Phi(0)}\left(-\nabla f\left(x_{\infty}\right)\right) \quad \text { a.e. on }\left[t_{*},+\infty[.\right.
$$

The continuity of $\dot{x}$ clearly shows that the previous inclusion holds for every $t \geq t_{*}$, which ends the proof.

4.2. Minorization by an exponential decay rate. Let us recall that the energylike function $E$ defined by $E(t):=\frac{1}{2}|\dot{x}(t)|^{2}+f(x(t))$ is decreasing along the trajectories of $(S)$ and that for almost every $t \in \mathbb{R}_{+}$,

$$
\dot{E}(t) \leq \Phi(0)-\Phi(\dot{x}(t)) \leq 0
$$

(see [1] for more details). Let us define the function $H$ by

$$
H(t)=E(t)-f\left(x_{\infty}\right)-\left\langle\nabla f\left(x_{\infty}\right), x(t)-x_{\infty}\right\rangle .
$$

The function $H$ is nonincreasing; indeed, from (4.3) we have:

$$
\dot{H}(t) \leq \Phi(0)-\Phi(\dot{x}(t))-\left\langle\nabla f\left(x_{\infty}\right), \dot{x}(t)\right\rangle .
$$

Since $-\nabla f\left(x_{\infty}\right) \in \partial \Phi(0)$, the subdifferential inequality gives $-\left\langle\nabla f\left(x_{\infty}\right), \dot{x}(t)\right\rangle \leq$ $\Phi(\dot{x}(t))-\Phi(0)$ and the announced result follows. The Lyapounov function $H$ will play an essential role throughout this section. The next result asserts that either the solutions to $(S)$ converge in a finite time or the convergence rate is minorized by some negative exponential. 
Theorem 4.2. Under hypotheses $(\mathcal{H})$, assume that $\partial \Phi(0)$ is a convex polyhedron and that (4.2) holds. Suppose moreover that there exist $\eta>0$ and $\alpha \geq 0$ such that

$$
|x| \leq \eta \quad \Longrightarrow \quad e(\partial \Phi(x), \partial \Phi(0)) \leq \alpha|x| .
$$

The function $f$ is assumed to be convex. Let $x$ be the unique solution of the differential inclusion $(S)$ and let us denote by $x_{\infty}:=\lim _{t \rightarrow+\infty} x(t)$ its limit when $t \rightarrow+\infty$. Then, one of the following cases holds:

(i) There exists $t_{0} \geq 0$ such that $x(t)=x_{\infty}$ for every $t \geq t_{0}$.

(ii) There exist $t_{1} \geq 0$ and $A>0$ such that

$$
\forall t \geq t_{1}, \quad \int_{t}^{+\infty}|\dot{x}(s)|^{2} d s \geq A e^{-2 \alpha t} .
$$

If moreover $\alpha=0$, then case $(i)$ necessarily holds, i.e. the solution $x$ of $(S)$ is stabilized in a finite time.

Proof. Consider the function $H$ defined above; we have for every $t \in \Gamma$,

$$
\dot{H}(t)=\left\langle\dot{x}(t), \ddot{x}(t)+\nabla f(x(t))-\nabla f\left(x_{\infty}\right)\right\rangle .
$$

For every $t \in \Gamma$, we have $-\ddot{x}(t)-\nabla f(x(t)) \in \partial \Phi(\dot{x}(t))$. Let us define $\xi(t)$ as the unique element of $\partial \Phi(0)$ such that

$$
d(-\ddot{x}(t)-\nabla f(x(t)), \partial \Phi(0))=|\xi(t)+\ddot{x}(t)+\nabla f(x(t))| .
$$

It is then clear that, for every $t \in \Gamma$,

$$
|\xi(t)+\ddot{x}(t)+\nabla f(x(t))| \leq \sup _{y \in \partial \Phi(\dot{x}(t))} d(y, \partial \Phi(0))=e(\partial \Phi(\dot{x}(t)), \partial \Phi(0)) .
$$

Since $\lim _{t \rightarrow+\infty} \dot{x}(t)=0$, there exists $t_{0} \geq 0$ such that $|\dot{x}(t)| \leq \eta$ for every $t \geq t_{0}$. Hence we deduce from assumption (4.5) and the previous inequality that

$$
\forall t \in\left[t_{0},+\infty[\cap \Gamma, \quad|\xi(t)+\ddot{x}(t)+\nabla f(x(t))| \leq \alpha|\dot{x}(t)| .\right.
$$

Since the set $\partial \Phi(0)$ is a convex polyhedron, we infer from Proposition 4.1 the existence of $t_{1} \geq t_{0}$ such that for every $t \geq t_{1}$, we have $\dot{x}(t) \in N_{\partial \Phi(0)}\left(-\nabla f\left(x_{\infty}\right)\right)$. Since $\xi(t) \in \partial \Phi(0)$, it ensues that

$$
\left\langle\dot{x}(t), \xi(t)+\nabla f\left(x_{\infty}\right)\right\rangle \leq 0 .
$$

In view of (4.6), (4.7) and (4.8), we conclude that

$$
\forall t \in\left[t_{1},+\infty\left[\cap \Gamma, \quad \dot{H}(t) \geq-\alpha|\dot{x}(t)|^{2} .\right.\right.
$$

Let us integrate the previous inequality on $[t,+\infty[$ to obtain:

$$
\frac{1}{2}|\dot{x}(t)|^{2}+f(x(t))-f\left(x_{\infty}\right)-\left\langle\nabla f\left(x_{\infty}\right), x(t)-x_{\infty}\right\rangle \leq \alpha \int_{t}^{+\infty}|\dot{x}(s)|^{2} d s .
$$

From the convexity inequality $f(x) \geq f\left(x_{\infty}\right)+\left\langle\nabla f\left(x_{\infty}\right), x-x_{\infty}\right\rangle$, we deduce that

$$
\forall t \geq t_{1}, \quad|\dot{x}(t)|^{2} \leq 2 \alpha \int_{t}^{+\infty}|\dot{x}(s)|^{2} d s .
$$

An immediate integration on $\left[t_{1}, t\right]$ shows that

$$
\forall t \geq t_{1}, \quad \int_{t}^{+\infty}|\dot{x}(s)|^{2} d s \geq\left(\int_{t_{1}}^{+\infty}|\dot{x}(s)|^{2} d s\right) e^{-2 \alpha\left(t-t_{1}\right)} .
$$


If $\int_{t_{1}}^{+\infty}|\dot{x}(s)|^{2} d s=0$, then clearly $|\dot{x}(t)|=0$ for every $t \geq t_{1}$. If $\int_{t_{1}}^{+\infty}|\dot{x}(s)|^{2} d s>0$, the expected formula is obtained by setting $\left.A:=\left(\int_{t_{1}}^{+\infty}|\dot{x}(s)|^{2} d s\right)\right) e^{2 \alpha t_{1}}$.

Now assume that $\alpha=0$. Let us argue by contradiction and assume that case (ii) holds, i.e. there exists $A>0$ such that $\int_{t}^{+\infty}|\dot{x}(s)|^{2} d s \geq A$ for $t$ large enough. This clearly contradicts the fact that $\lim _{t \rightarrow+\infty} \int_{t}^{+\infty}|\dot{x}(s)|^{2} d s=0$, and we conclude that $x(t)=x_{\infty}$ for $t$ large enough.

In the next corollary, we particularize the setting of Theorem 4.2 to the case $\Phi:=\sigma_{C}+\Psi$, for some convex set $C \subset \mathbb{R}^{n}$ and some smooth convex function $\Psi: \mathbb{R}^{n} \rightarrow \mathbb{R}$.

Corollary 4.3. Let $C$ be a closed convex polyhedron of $\mathbb{R}^{n}$ which is bounded and such that $0 \in \operatorname{int}(C)$. Consider a convex function $\Psi: \mathbb{R}^{n} \rightarrow \mathbb{R}$ of class $\mathcal{C}^{1}$ such that there exist $\eta>0$ and $\alpha \geq 0$ satisfying

$$
|x| \leq \eta \quad \Longrightarrow \quad|\nabla \Psi(x)| \leq \alpha|x| .
$$

Assume that the function $\Phi: \mathbb{R}^{n} \rightarrow \mathbb{R}$ is defined by $\Phi:=\sigma_{C}+\Psi$. Suppose moreover that the function $f: \mathbb{R}^{n} \rightarrow \mathbb{R}$ is convex and verifies $\left(\mathcal{H}_{f}-i, i i\right)$. Then the solution $x$ to $(S)$ satisfies the same conclusions as in Theorem 4.2 .

Proof. Immediate from the previous theorem. See also the proof of Corollary 3.3.

The balls $\mathbb{B}_{1}$ and $\mathbb{B}_{\infty}$ (cf. Examples 2.4 and 2.5 ) provide us with two standard examples of sets $C$ satisfying the assumptions of Corollary 4.3.

Assume now that the function $\Psi$ equals zero, i.e. the friction has no viscous component. It is then immediate from Corollary 4.3 that every solution to $(S)$ stops after a finite time. Therefore the result of finite time stabilization obtained in [5] appears as a consequence of Corollary 4.3. By comparison with Corollary 3.4, one can wonder if there exists a critical coefficient $b_{c}$ such that $b \in\left[0, b_{c}\right.$ [ implies the finite time convergence of the trajectory. This question, which is implicitly contained in [11], is still an open problem.

4.3. Majorization by an exponential decay rate. In the previous paragraph, we have shown that either the solutions to $(S)$ converge in a finite time or the convergence rate is minorized by some negative exponential. Conversely, we are going to prove that under adequate conditions the convergence rate is majorized by some negative exponential. The key assumption of the next theorem is the existence of a symmetric positive definite matrix $L \in \mathcal{M}_{n}(\mathbb{R})$ such that

$$
e\left(\partial \Phi(x), \partial\left[\Phi^{\prime}(0 ; .)\right](x)+L x\right)=O\left(|x|^{2}\right) \quad \text { when } x \rightarrow 0 .
$$

This condition is clearly stronger than (4.2). Suppose that the function $\Phi$ equals $\Phi:=\sigma_{C}+\Psi$ for some convex set $C \subset \mathbb{R}^{n}$ and some convex function $\Psi: \mathbb{R}^{n} \rightarrow \mathbb{R}$ of class $\mathcal{C}^{3}$ such that $\nabla \Psi(0)=0$. In this case, equality (4.9) is satisfied with $L:=\nabla^{2} \Psi(0)$. This can be easily obtained from a second-order Taylor expansion of the function $\nabla \Psi$ in the neighborhood of 0 .

Theorem 4.4. Under the hypotheses $(\mathcal{H})$, suppose that the set $\partial \Phi(0)$ is a convex polyhedron and that there exists a symmetric positive definite matrix $L \in \mathcal{M}_{n}(\mathbb{R})$ 
satisfying (4.9). Assume that the function $f$ is strongly convex, i.e. there exists $M>0$ such that

$$
\forall x, y \in \mathbb{R}^{n}, \quad f(x) \geq f(y)+\langle\nabla f(y), x-y\rangle+M / 2|x-y|^{2} .
$$

Let $x$ be the unique solution of the differential inclusion $(S)$ and let us denote by $x_{\infty}:=\lim _{t \rightarrow+\infty} x(t)$ its limit when $t \rightarrow+\infty$. Then there exist $C, \gamma>0$ and $t_{0} \geq 0$ such that

$$
\forall t \geq t_{0}, \quad|\dot{x}(t)| \leq C e^{-\gamma t} .
$$

Denoting by $\lambda_{1}$ (resp. $\lambda_{n}$ ) the smallest (resp. largest) eigenvalue of the matrix $L$, any positive exponent $\gamma$ such that $\gamma<\min \left\{\lambda_{1} / 6,\left(\sqrt{\lambda_{n}^{2}+4 M}-\lambda_{n}\right) / 8\right\}$ satisfies the previous estimate.

Proof. For every $t \in \Gamma$, we have $-\ddot{x}(t)-\nabla f(x(t)) \in \partial \Phi(\dot{x}(t))$. Let us define $\xi(t)$ as the unique element of the set $\partial\left[\Phi^{\prime}(0 ;).\right](\dot{x}(t))+L \dot{x}(t)$ such that

$$
d\left(-\ddot{x}(t)-\nabla f(x(t)), \partial\left[\Phi^{\prime}(0 ; .)\right](\dot{x}(t))+L \dot{x}(t)\right)=|\xi(t)+\ddot{x}(t)+\nabla f(x(t))| .
$$

In view of assumption (4.9) we have, for every $t \in \Gamma$,

$$
|\xi(t)+\ddot{x}(t)+\nabla f(x(t))| \leq e\left(\partial \Phi(\dot{x}(t)), \partial\left[\Phi^{\prime}(0 ; .)\right](\dot{x}(t))+L \dot{x}(t)\right)=O\left(|\dot{x}(t)|^{2}\right) .
$$

Let us define the auxiliary function $F$ by:

$$
F(t):=\left\langle\dot{x}(t), x(t)-x_{\infty}\right\rangle+\frac{1}{2}\left\langle L\left(x(t)-x_{\infty}\right), x(t)-x_{\infty}\right\rangle .
$$

An elementary computation shows that for every $t \in \Gamma$

$$
\begin{aligned}
\dot{F}(t)=|\dot{x}(t)|^{2} & +\left\langle\ddot{x}(t)+L \dot{x}(t), x(t)-x_{\infty}\right\rangle \\
=|\dot{x}(t)|^{2} & +\left\langle\ddot{x}(t)+\xi(t)+\nabla f(x(t)), x(t)-x_{\infty}\right\rangle \\
& -\left\langle\xi(t)-L \dot{x}(t)+\nabla f\left(x_{\infty}\right), x(t)-x_{\infty}\right\rangle \\
& -\left\langle\nabla f(x(t)), x(t)-x_{\infty}\right\rangle+\left\langle\nabla f\left(x_{\infty}\right), x(t)-x_{\infty}\right\rangle .
\end{aligned}
$$

Let us fix some $\eta \in] 0, \lambda_{1}$ [. Since $\lim _{t \rightarrow+\infty} x(t)=x_{\infty}$, we obtain in view of inequality (4.11) that there exists $t_{1} \geq 0$ such that, for every $t \in\left[t_{1},+\infty[\cap \Gamma\right.$,

$$
\left|\left\langle\ddot{x}(t)+\xi(t)+\nabla f(x(t)), x(t)-x_{\infty}\right\rangle\right| \leq \eta|\dot{x}(t)|^{2} .
$$

From the definition of $\xi(t)$, we have for every $t \in \Gamma$

$$
\xi(t)-L \dot{x}(t) \in \partial\left[\Phi^{\prime}(0 ; .)\right](\dot{x}(t)) \subset \partial \Phi(0) .
$$

Since the set $\partial \Phi(0)$ is a convex polyhedron, we infer from Proposition 4.1 the existence of $t_{2} \geq t_{1}$ such that $\dot{x}(t) \in N_{\partial \Phi(0)}\left(-\nabla f\left(x_{\infty}\right)\right)$ for every $t \geq t_{2}$. An immediate integration on $\left[t,+\infty\right.$ [ shows that $x(t)-x_{\infty} \in-N_{\partial \Phi(0)}\left(-\nabla f\left(x_{\infty}\right)\right)$ for every $t \geq t_{2}$. In view of (4.14), this implies that, for every $t \in\left[t_{2},+\infty[\cap \Gamma\right.$,

$$
\left\langle\xi(t)-L \dot{x}(t)+\nabla f\left(x_{\infty}\right), x(t)-x_{\infty}\right\rangle \geq 0 .
$$

On the other hand, the convexity of $f$ gives

$$
\left\langle\nabla f(x(t)), x(t)-x_{\infty}\right\rangle \geq f(x(t))-f\left(x_{\infty}\right) .
$$

By combining (4.12), (4.13), (4.15) and (4.16), we find

$\forall t \in\left[t_{2},+\infty\left[\cap \Gamma, \dot{F}(t) \leq(1+\eta)|\dot{x}(t)|^{2}-\left[f(x(t))-f\left(x_{\infty}\right)-\left\langle\nabla f\left(x_{\infty}\right), x(t)-x_{\infty}\right\rangle\right]\right.\right.$. 
By introducing the function $H$ defined by (4.4), the previous inequality can be rewritten as

$$
\forall t \in\left[t_{2},+\infty\left[\cap \Gamma, \quad \dot{F}(t)+H(t) \leq(3 / 2+\eta)|\dot{x}(t)|^{2} .\right.\right.
$$

Let us now differentiate the function $H$; we find for every $t \in \Gamma$

$$
\begin{aligned}
\dot{H}(t)= & \langle\ddot{x}(t), \dot{x}(t)\rangle+\langle\nabla f(x(t)), \dot{x}(t)\rangle-\left\langle\nabla f\left(x_{\infty}\right), \dot{x}(t)\right\rangle \\
= & \langle\ddot{x}(t)+\nabla f(x(t))+\xi(t), \dot{x}(t)\rangle+\left\langle-\nabla f\left(x_{\infty}\right)-\xi(t)+L \dot{x}(t), \dot{x}(t)\right\rangle \\
& -\langle L \dot{x}(t), \dot{x}(t)\rangle .
\end{aligned}
$$

In view of (4.11), we have

$$
|\langle\ddot{x}(t)+\nabla f(x(t))+\xi(t), \dot{x}(t)\rangle|=O\left(|\dot{x}(t)|^{3}\right) \quad \text { when } t \rightarrow+\infty .
$$

From the definition of $\xi(t)$, we have $\dot{x}(t) \in N_{\partial \Phi(0)}(\xi(t)-L \dot{x}(t))$ for every $t \in \Gamma$. Since $-\nabla f\left(x_{\infty}\right) \in \partial \Phi(0)$, we infer that

$$
\left\langle-\nabla f\left(x_{\infty}\right)-\xi(t)+L \dot{x}(t), \dot{x}(t)\right\rangle \leq 0 .
$$

From (4.18), (4.19) and (4.20), we conclude that

$$
\dot{H}(t) \leq-\langle L \dot{x}(t), \dot{x}(t)\rangle+O\left(|\dot{x}(t)|^{3}\right) .
$$

Hence there exists $t_{3} \geq t_{2}$ such that for every $t \in\left[t_{3},+\infty[\cap \Gamma\right.$,

$$
\dot{H}(t) \leq-\left(\lambda_{1}-\eta\right)|\dot{x}(t)|^{2} .
$$

Let us multiply (4.17) by $A_{\eta}:=\left(\lambda_{1}-\eta\right) /(3 / 2+\eta)$ and add to (4.21); we obtain

$$
\dot{H}(t)+A_{\eta} \dot{F}(t)+A_{\eta} H(t) \leq 0 .
$$

Our purpose now is to deduce from (4.22) a differential equation involving a single function. This is made possible owing to the following relations between the functions $F$ and $H$ :

$$
\forall t \geq 0, \quad F(t) \geq-H(t) / \lambda_{1} \quad \text { and } \quad H(t) \geq B F(t),
$$

where $B$ is a positive real that we are going to determine. We classically have, for all $\theta>0$,

$$
\left|\left\langle\dot{x}(t), x(t)-x_{\infty}\right\rangle\right| \leq \frac{|\dot{x}(t)|^{2}}{2 \theta}+\frac{\theta}{2}\left|x(t)-x_{\infty}\right|^{2},
$$

and hence

$$
-\frac{|\dot{x}(t)|^{2}}{2 \theta}+\frac{-\theta+\lambda_{1}}{2}\left|x(t)-x_{\infty}\right|^{2} \leq F(t) \leq \frac{|\dot{x}(t)|^{2}}{2 \theta}+\frac{\theta+\lambda_{n}}{2}\left|x(t)-x_{\infty}\right|^{2} .
$$

Taking $\theta=\lambda_{1}$ in the first inequality of (4.24), we obtain $F(t) \geq-|\dot{x}(t)|^{2} /\left(2 \lambda_{1}\right) \geq$ $-H(t) / \lambda_{1}$, which is the first inequality of (4.23). On the other hand, from the strong convexity assumption (4.10), we have

$$
H(t) \geq \frac{1}{2}|\dot{x}(t)|^{2}+\frac{M}{2}\left|x(t)-x_{\infty}\right|^{2} .
$$

Setting $\tau(\theta):=\min \left\{\theta, M /\left(\theta+\lambda_{n}\right)\right\}$, we deduce from the second inequality of (4.24) and (4.25) that

$$
H(t) \geq \tau(\theta) F(t)
$$

We let the reader check that the function $\tau: \mathbb{R}_{+} \rightarrow \mathbb{R}$ achieves its maximum at $B:=\left(\sqrt{\lambda_{n}^{2}+4 M}-\lambda_{n}\right) / 2$ and that $\tau(B)=B$. Taking $\theta=B$ in inequality (4.26), 
we obtain the second inequality of (4.23). We deduce from (4.22) and the second inequality of (4.23) that

$$
\dot{H}(t)+A_{\eta} \dot{F}(t)+A_{\eta} B F(t) \leq 0 .
$$

Let us multiply (4.22) by $B$ and (4.27) by $A_{\eta}$; adding the two inequalities and setting $G(t):=H(t)+A_{\eta} F(t)$, this yields:

$$
\forall t \in\left[t_{3},+\infty\left[\cap \Gamma, \quad\left(A_{\eta}+B\right) \dot{G}(t)+A_{\eta} B G(t) \leq 0 .\right.\right.
$$

An elementary integration on $\left[t_{3}, t\right]$ gives:

$$
\forall t \in\left[t_{3},+\infty\left[, \quad G(t) \leq G\left(t_{3}\right) e^{-\frac{A_{\eta} B}{A_{\eta}+B}\left(t-t_{3}\right)} .\right.\right.
$$

From the first inequality of (4.23), we have $G(t) \geq H(t)-A_{\eta} / \lambda_{1} H(t)$ and since $A_{\eta} \leq 2 \lambda_{1} / 3$, we finally obtain $G(t) \geq H(t) / 3$. Setting $C:=3 G\left(t_{3}\right) e^{\frac{A_{\eta} B}{A_{\eta}+B} t_{3}}$, we deduce in view of (4.28) that

$$
\forall t \in\left[t_{3},+\infty\left[, \quad H(t) \leq C e^{-\frac{A_{\eta} B}{A_{\eta}+B} t} .\right.\right.
$$

Since $\frac{1}{2}|\dot{x}(t)|^{2} \leq H(t)$, we conclude that

$$
\forall t \in\left[t_{3},+\infty\left[, \quad|\dot{x}(t)| \leq \sqrt{2 C} e^{-\frac{1}{2} \frac{A_{\eta} B}{A_{\eta}+B} t} .\right.\right.
$$

Now consider some exponent $\gamma>0$ such that $\gamma<\min \left\{\lambda_{1} / 6, B / 4\right\}$. Remark that

$$
\lim _{\eta \rightarrow 0} \frac{A_{\eta} B}{A_{\eta}+B}=\frac{\frac{2 \lambda_{1}}{3} B}{\frac{2 \lambda_{1}}{3}+B} \geq \frac{1}{2} \min \left\{\frac{2 \lambda_{1}}{3}, B\right\}>2 \gamma .
$$

By choosing $\eta$ small enough so as to have $A_{\eta} B /\left(A_{\eta}+B\right) \geq 2 \gamma$, inequality (4.29) gives $|\dot{x}(t)| \leq \sqrt{2 C} e^{-\gamma t}$ for every $t \geq t_{3}$.

Let us notice that the key condition (4.9) of Theorem 4.4 entails condition (4.5) of Theorem 4.2. This remark gives rise to the following corollary.

Corollary 4.5. Under the assumptions of Theorem 4.4, one of the following cases holds:

(i) There exists $t_{0} \geq 0$ such that $x(t)=x_{\infty}$ for every $t \geq t_{0}$.

(ii) There exist $t_{1} \geq 0$ and $C_{1}, C_{2}, \gamma_{1}, \gamma_{2}>0$ such that

$$
\forall t \geq t_{1}, \quad|\dot{x}(t)| \leq C_{1} e^{-\gamma_{1} t} \quad \text { and } \quad \int_{t}^{+\infty}|\dot{x}(s)|^{2} d s \geq C_{2} e^{-\gamma_{2} t} .
$$

Any positive exponent $\gamma_{1}$ (resp. $\left.\gamma_{2}\right)$ such that $\gamma_{1}<\min \left\{\lambda_{1} / 6,\left(\sqrt{\lambda_{n}^{2}+4 M}-\lambda_{n}\right) / 8\right\}$ (resp. $\gamma_{2}>2 \lambda_{n}$ ) satisfies the previous estimate.

Proof. The first inequality of (4.30) immediately results from Theorem 4.4. Since $\partial\left[\Phi^{\prime}(0 ;).\right](x) \subset \partial \Phi(0)$ for every $x \in \mathbb{R}^{n}$, we have

$$
e(\partial \Phi(x), \partial \Phi(0)) \leq e\left(\partial \Phi(x), \partial\left[\Phi^{\prime}(0 ; .)\right](x)\right) .
$$

For every $y \in \partial \Phi(x)$, we have $d\left(y, \partial\left[\Phi^{\prime}(0 ;).\right](x)\right) \leq|L x|+d\left(y, \partial\left[\Phi^{\prime}(0 ;).\right](x)+L x\right)$, and taking the supremum when $y \in \partial \Phi(x)$, we infer that

$$
e\left(\partial \Phi(x), \partial\left[\Phi^{\prime}(0 ; .)\right](x)\right) \leq|L x|+e\left(\partial \Phi(x), \partial\left[\Phi^{\prime}(0 ; .)\right](x)+L x\right) .
$$


In view of condition (4.9), inequalities (4.31), (4.32) and the fact that $|L x| \leq \lambda_{n}|x|$ for every $x \in \mathbb{R}^{n}$, we conclude that

$$
e(\partial \Phi(x), \partial \Phi(0)) \leq \lambda_{n}|x|+O\left(|x|^{2}\right) .
$$

Hence condition (4.5) of Theorem 4.2 is satisfied with $\alpha:=\lambda_{n}+\varepsilon / 2$ for any $\varepsilon>0$. We deduce that either the solution $x$ of $(S)$ converges in a finite time or $\int_{t}^{+\infty}|\dot{x}(s)|^{2} d s \geq C_{2} e^{-\left(2 \lambda_{n}+\varepsilon\right) t}$ for some positive $C_{2}$ and $t$ large enough.

\section{ACKNOWLEDGMEnTs}

I am grateful to Professors J.I. Díaz and V. Millot who suggested to me the reading of their heuristic paper [11].

\section{REFERENCES}

1. S. Adly, H. Attouch and A. Cabot, Finite time stabilization of nonlinear oscillators subject to dry friction, Progresses in Nonsmooth Mechanics and Analysis (edited by P. Alart, O. Maisonneuve and R.T. Rockafellar), Advances in Mathematics and Mechanics, Kluwer, pp. 289-304. MR2205459 (2006i:70026)

2. S. Adly and D. Goeleven, A stability theory for second order nonsmooth dynamical systems with application to friction problems, Journal de Mathématiques Pures et Appliquées 83 (2004), 17-51. MR2023053 (2004j:34029)

3. H. Amann and J. I. Díaz, A note on the dynamics of an oscillator in the presence of strong friction, Nonlinear Analysis 55 (2003), 209-216. MR2007469 (2004g:34046)

4. H. Attouch, X. Goudou and P. Redont, The heavy ball with friction method. I The continuous dynamical system, Communications in Contemporary Math 2 (2000), 1-34. MR1753136 (2001b:37025)

5. A. Bamberger and H. Cabannes, Mouvement d'une corde vibrante soumise à un frottement solide, C. R. Acad. Sci. Paris Sér. I Math. 292 (1981), 699-702. MR0618890 (82h:73051)

6. H. Brézis, Opérateurs maximaux monotones et semi-groupes de contractions dans les espaces de Hilbert, Math. Studies 5, North Holland, Amsterdam (1973). MR0348562 (50:1060)

7. B. Brogliato, Nonsmooth Mechanics, Springer CCES, 2nd edition, London (1999).

8. H. Cabannes, Mouvement d'une corde vibrante soumise à un frottement solide, C. R. Acad. Sci. Paris Sér. A-B 287 (1978), 671-673. MR0514553 (83m:73056)

9. H. Cabannes, Study of motions of a vibrating string subject to solid friction, Math. Methods Appl. Sci. 3 (1981), 287-300. MR0657297 (83e:73049)

10. J. I. Díaz and A. Liñán, On the asymptotic behavior of a damped oscillator under a sublinear friction term, Rev. R. Acad. Cien. Serie A. Mat. 95 (2001), 155-160. MR1899359 (2003c:34005)

11. J. I. Díaz and V. Millot, Coulomb friction and oscillation: stabilization in finite time for a system of damped oscillators, XVIII CEDYA: Congress on Differential Equations and Applications/VIII CMA: Congress on Applied Mathematics (Tarragona, 2003).

12. D. Goeleven, D. Motreanu, Y. Dumont and M. Rochdi, Variational and Hemivariational Inequalities, Volume 1: Unilateral Analysis and Mechanics, Kluwer Academic Publishers, Boston, 2003. MR2006373 (2004g:49004)

13. D.W. Jordan and P. Smith, Nonlinear ordinary differential equations, Second Edition, Oxford Applied Mathematics and Computing Science Series, The Clarendon Press, Oxford University Press, New York (1987). MR0899734 (89a:34001)

14. M.D.P. Monteiro Marques, Differential inclusions in nonsmooth mechanical problems, Progress in nonlinear differential equations and their applications, 9, Birkhaüser (1993). MR1231975 (94g:34003)

15. J.J. Moreau, Dynamique de systèmes à liaisons unilatérales avec frottement sec éventuel: essais numériques, LMGC, Montpellier, Note Technique $\mathrm{n}^{\circ}$ 85-1 (1985).

16. J.J. Moreau, Une formulation du contact à frottement sec; application au calcul numérique, C. R. Acad. Sci. Paris Sér. II 302 (1986), 799-801. MR0977371 (89k:73008)

17. P.D. Panagiotopoulos, Inequality problems in Mechanics and Applications, Birkhaüser, Boston (1985). MR0896909 (88h:49003) 
18. Y. Renard, Modélisation des Instabilités liées au Frottement sec des Solides Elastiques, Aspect Théorique, Ph.D. Thesis, Grenoble I University (1998).

19. R.T. Rockafellar and R. Wets, Variational analysis, Springer, Berlin (1998). MR1491362 (98m:49001)

Université de Limoges, 123 avenue Albert Thomas, 87060 Limoges Cedex, France

E-mail address: alexandre.cabot@unilim.fr 